\title{
Towards the Formalization of Fractional Calculus in Higher-Order Logic ${ }^{\star}$
}

\author{
Umair Siddique*, Osman Hasan**, and Sofiène Tahar* \\ *Department of Electrical and Computer Engineering, \\ Concordia University, Montreal, Quebec, Canada \\ ${ }^{* *}$ School of Electrical Engineering and Computer Science, \\ National University of Sciences and Technology, Islamabad, Pakistan \\ \{muh_sidd, tahar\}@ece. concordia.ca \\ osman.hasan@seecs.nust.edu.pk \\ http://save.seecs.nust.edu.pk/projects/fc.html
}

\begin{abstract}
Fractional calculus is a generalization of classical theories of integration and differentiation to arbitrary order (i.e., real or complex numbers). In the last two decades, this new mathematical modeling approach has been widely used to analyze a wide class of physical systems in various fields of science and engineering. In this paper, we describe an ongoing project which aims at formalizing the basic theories of fractional calculus in the HOL Light theorem prover. Mainly, we present the motivation and application of such formalization efforts, a roadmap to achieve our goals, current status of the project and future milestones.
\end{abstract}

Keywords: Fractional Calculus, Higher-Order Logic, Theorem Proving

\section{Motivation and Background}

Physical and engineering systems are classified as continuous, discrete or hybrid depending upon the nature of underlying system parameters. The rich theories of mathematics provide the necessary tools to study the behaviour of such systems ranging from very small biological organisms to the modern Quantum mechanical phenomenons. Generally, differential equations [39] and difference equations [12] are used to characterize the dynamics of these systems. Consequently, the concept of higher-order differentiation and integration are widely studied in diverse disciplines of science and engineering. For example, it is well understood that the first derivative $\left(\frac{d}{d t} f(t)\right)$ and second derivative $\left(\frac{d^{2}}{d t^{2}} f(t)\right)$ of a function describe the rate of change and measure of concavity, respectively. However, we rarely think what if the order $(n)$ of higher-order derivative $\left(\frac{d^{n}}{d t^{n}}\right)$ becomes a real, complex or an irrational number? One immediate question arises in our minds is the existence or possibility of such a concept in mathematics. Interestingly, this seemingly new concept dates back to 1695 when L'Hôpital asked Leibniz

\footnotetext{
* This is authors' version of CICM-2015 paper. The final publication is available at http://link.springer.com
} 
regarding his notation $\frac{d^{n} y}{d x^{n}}$ : "what if $n$ is $\frac{1}{2}$ ". In reply, Leibniz [20] prophesied in his letter, "... Thus it follows that $d^{\frac{1}{2}} x$ will be equal to $x \sqrt{d x: x}$. This is an apparent paradox from which, one day, useful consequences can be drawn ...". Leibniz's initial work on the problem of defining the derivative of arbitrary order gave birth to a new field of research in mathematics (called fractional calculus) and attracted the attention of many physicists, engineers and geometers. Some of the great mathematicians and physicists who touched the field of fractional calculus are Riemann, Liouville, Laurent, Heaviside and Riesz [24].

The concept of fractional calculus has great potential to change the way we model and analyze the systems. It provides good opportunity to scientists and engineers for revisiting the origins. We briefly outline some of the the main applications of fractional calculus in Table 1 . The importance of fractional calculus can be realized by the following quote from Miller and Ross [24. They stated:

“... The fractional calculus finds use in many fields of science and engineering, including fluid flow, rheology, diffusive transport akin to diffusion, electrical networks, electromagnetic theory, and probability.... It seems that hardly a field of science or engineering has remained untouched by this topic..."

\begin{tabular}{|c|c|}
\hline Field & Applications \\
\hline Control Engineering & $\begin{array}{l}\text { - System identification }[17] \\
\text { - Biomimetic (bionics) control }[7] \\
\text { - Trajectory control }[1] \\
\text { - Temperature control }[30] \\
\text { - Fractional } P I^{\alpha} \text { controller }[23]\end{array}$ \\
\hline Signal Processing & $\begin{array}{l}\text { - Fractional order integrator }[19] \\
\text { - Fractional order FIR differentiator } 37] \\
\text { - IIR-type fractional order differentiator } 38 \\
\text { - Modeling of speech signals } 18\end{array}$ \\
\hline Image Processing & $\begin{array}{l}\text { - Image restoration and edge detection }[29 \\
\text { - Satellite image classification } 6\end{array}$ \\
\hline Electromagnetics & $\begin{array}{l}\text { - Fractional curl operators }[1325] \\
\text { - Fractional Rectangular waveguides }[14\end{array}$ \\
\hline Communication & $\begin{array}{l}\text { - Secure chaotic communication }[1] \\
\text { - Informational network traffic modeling }[40\end{array}$ \\
\hline Biology & $\begin{array}{l}\text { - Neuron modeling }[5] \\
\text { - Biophysical processes }[8] \\
\text { - Modeling of complex dynamics of tissues }[22\end{array}$ \\
\hline
\end{tabular}

Table 1: Applications of Fractional Calculus

Nowadays engineering systems exhibiting fractional order dynamics are increasingly used in some safety-critical applications such as control systems, signal processing, electromagnetics and electrical networks (as listed in Table 1). 
For example, fractional meta-materials based devices are used to build sensitive military and defence equipments and electromagnetic stealth technology [21. Considering these facts, it is quite interesting and important to build a logical reasoning framework which can be used to formally verify such sophisticated applications within the sound core of a proof assistant. In fact, proof assistants have been successfully used to formalize and verify some challenging and paradoxical mathematical results, e.g., the formal proofs of the Kepler Conjecture (Flyspeck project) [16] and the Odd Order Theorem [15].

In this paper, we present details of an ongoing project ${ }^{1}$ to develop a formal reasoning support for fractional calculus in higher-order-logic theorem prover. This project was originally started at the System Analysis and Verification (SAVe) lab ${ }^{2}$ in 2010. Earlier formalization was done in the HOL4 theorem prover with the main focus on fractional operators for real-valued functions and the verification of fractional order electrical components. Later on, the scope of the project was expanded to formalize fractional calculus involving complex-valued functions due to its various engineering applications (as listed in Table 1). Currently, we are using the HOL Light theorem prover due to the availability of rich multivariate analysis libraries including Harrison's recent formalization of complex-valued Gamma function ${ }^{3}$ as well as the interesting related projects like Flyspeck [16] and the formalization of optics theories (i.e., ray, wave, electromagnetic and quantum) [2].

The rest of the paper is organized as follows: In Section 2 , we briefly review some commonly used notations and definitions of fractional order operators. We provide an outline of the proposed formalization framework in Section 3 . Consequently, the current status of the formalization and future milestones are discussed in Section 4. Finally, we conclude the paper in Section 5

\section{Mathematical Framework of Fractional Calculus}

There are different notations available for fractional derivatives and integrals. We use $J_{a}^{v} f(x)$ and $D^{v} f(x)$ for fractional integral and fractional derivative, respectively. In these notations, $v$ is the order of integration or differentiation and $a$ is the lower limit of integration.

For every function $(f: \mathbb{C} \rightarrow \mathbb{C})$; and for every number $v \in \mathbb{R}$ or $\mathbb{C}, J_{a}^{v}$ and $D^{v}$ should be related to $f$ by the following criteria [10].

1. If $f(x)$ is an analytic function, then $J_{a}^{v} f(x)$ and $D^{v} f(x)$ must also be an analytic function of the variable $x$ and of the order $v$ of integration or differentiation.

2. The operations $J_{a}^{v} f(x)$ and $D^{v} f(x)$ must produce the same result as ordinary integration/differentiation when $v$ is a positive integer.

\footnotetext{
1 http://save.seecs.nust.edu.pk/projects/fc.html

2 http://save.seecs.nust.edu.pk

3 https://code.google.com/p/hol-light/source/browse/trunk/Multivariate/ gamma.ml
} 
3. The fractional operators must be linear.

$$
\begin{gathered}
J_{a}^{v}[\alpha f(x)+\beta g(x)]=\alpha J_{a}^{v} f(x)+\beta J_{a}^{v} g(x) \\
D^{v}[\alpha f(x)+\beta g(x)]=\alpha D^{v} f(x)+\beta D^{v} g(x)
\end{gathered}
$$

4. The operation of order zero must leave the function unchanged.

$$
J_{a}^{0} f=f \quad \text { and } \quad D^{0} f=f
$$

5. The law of exponents must hold for integration and differentiation of arbitrary order under sufficient conditions on function $f$.

$$
J_{a}^{u}\left(J_{a}^{v} f\right)=J_{a}^{u+v} f \quad \text { and } \quad D^{u}\left(D^{v} f\right)=D^{u+v} f
$$

Fractional integrals and fractional derivatives are also referred to as Differintegrals [27] and there are more than ten well-known definitions for Differintegrals 9. We describe here two of them, which are most widely used in analyzing real-world problems. These are the Riemann-Liouville and Grünwald-Letnikov definitions, which are also equivalent for a wide class of functions [31].

\section{Riemann-Liouville (RL) Definition:}

$$
J_{a}^{v} f(x)=\frac{1}{\Gamma(v)} \int_{a}^{x}(x-t)^{v-1} f(t) d t
$$

where $J_{a}^{v} f(x)$ represents fractional integration with order $v$ and lower integration limit $a$. The parameter $a=0$ gives the Riemann definition and $a=-\infty$ gives the Liouville definition of fractional integration. Indeed Equation (5) is the generalization of Cauchy's repeated integration formula to non-integer $v$ [32]. Where $\Gamma($.$) in the above definition denotes the Gamma function which is defined using$ the well-known improper integral as follows:

$$
\Gamma(z)=\int_{0}^{\infty} t^{z-1} e^{-t} d t
$$

for $\operatorname{Re}(z)>0$.

The fractional differentiation is given as follows:

$$
D^{v} f(x)=\left(\frac{d}{d x}\right)^{m} J_{a}^{m-v} f(x)
$$

where $m$ represents the ceiling of $v$, i.e., $\lceil v\rceil$.

\section{Grünwald-Letnikov (GL) Definition:}

$$
{ }_{c} D_{x}^{v} f(x)=\lim _{h \rightarrow 0} h^{-v} \sum_{k=0}^{\left[\frac{x-c}{h}\right]}(-1)^{k}\left(\begin{array}{l}
v \\
k
\end{array}\right) f(x-k h)
$$

Grünwald-Letnikov definition caters for both fractional differentiation and integration, as positive values of $v$ give fractional differentiation and negative values of $v$ give fractional integration. Here, $\left(\begin{array}{l}v \\ k\end{array}\right)$ represents the binomial coefficients, which are described in terms of the Gamma function. 


\section{Formal Analysis Framework}

The proposed framework, given in Figure 1, outlines the main ideas and roadmap to formalize the basic theory behind fractional calculus. The whole framework

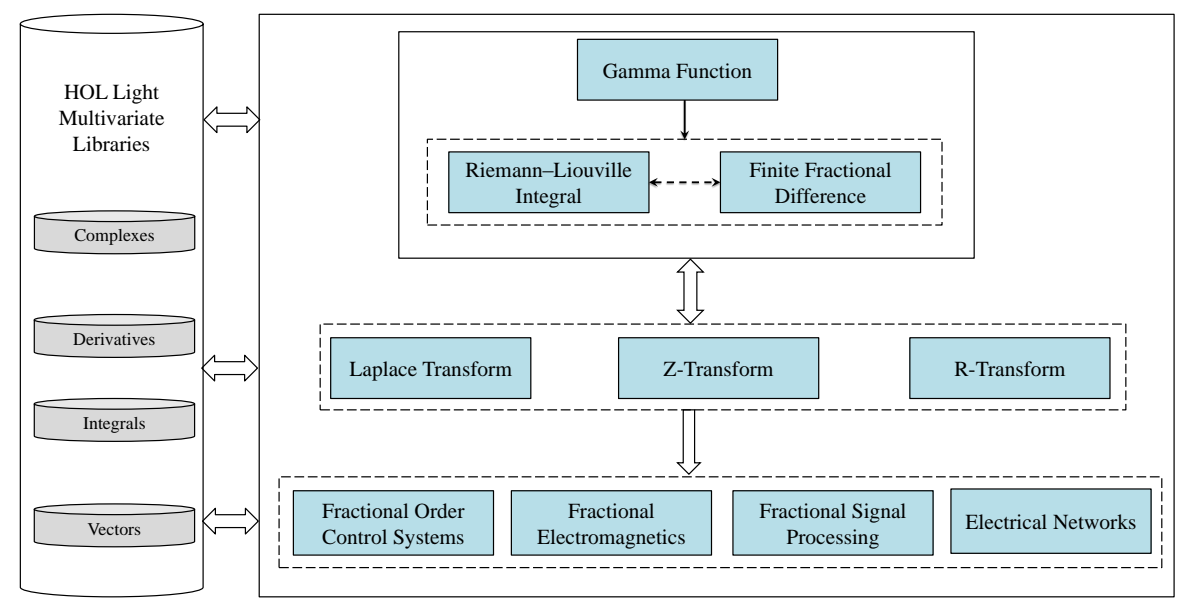

Fig. 1. Formalization Framework for Fractional Calculus

can be decomposed mainly into three major parts which are the formalization of the core definitions of fractional order operators, formalization of supporting transformations (i.e., Laplace transform [26, Z-transform [28] and R-Transform 3]) and engineering applications. The first part heavily relies upon the Gamma function as mentioned in Section 2. So the core step is to formalize the Gamma function in higher-order logic (HOL) and verify its important properties. Consequently, any definition of fractional order operators can be formalized in HOL. However, our focus is two main definitions, i.e., Riemann-Liouville (RL) and fractional difference which indeed represent continuous and discrete versions of fractional order operators, respectively. This step also involves the validation of all the properties mentioned in Section 2. This requires some important results of multivariate calculus such as the notion of Lebesgue measurability and Fubini's theorem which provides the reasoning support for iterated and double integrals. Interestingly, both of these requirements are available in the multivariate analysis libraries of HOL Light. The second step is the formalization of important integral transforms which are necessary to analytically solve linear fractional differential and difference equations. We mainly focus on three transforms, namely the Laplace transform, the Z-transform, and the recently introduced R-transform [4. All of these transformations are used to transform complicated fractional differential (or difference) equations to algebraic equations which are easier to manipulate and to deduce interesting properties. Building 
upon these fundamentals, our ultimate goal is to formally verify a variety of engineering systems including control systems, signal processing, electromagnetics and electrical networks. All formalization steps make use of different multivariate theories of HOL Light, e.g., derivatives, integrals, complex vectors and measure spaces. Finally, the developed libraries of this project will become part of the existing HOL Light libraries.

\section{Current Status and Future Milestones}

As mentioned earlier, the project initially considered only real-order fractional operators in the HOL4 theorem prover. The main difficulty was the little support to handle improper integrals which was required to formalize the real-valued Gamma function. Therefore, we extended the integration theory of HOL4 by formalizing a variant of improper integrals using sequential limits. This was then used to formalize the Gamma function and verify some of its main properties, such as the pseudo-recurrence relation $(\Gamma(z+1)=z \Gamma(z))$, the functional equation $(\Gamma(1)=1)$ and the factorial generalization $(\Gamma(k+1)=k !)$ 34. We utilized these foundations to formalize Differintegrals, given in Equations (5) and (7), which in turn can be used to represent the dynamics of fractional order systems in higher-order logic. We also verified theorems corresponding to some commonly used properties of Differintegrals namely Identity and Linearity. Consequently, we conducted the formal analysis of a fractional order electrical component namely resistoductor, a fractional integrator and a fractional differentiator circuit [33. Later on, the scope of the project was revised to include complex-valued functions and complex order fractional operators in HOL Light. The main requirement was to formalize the complex-valued Gamma function, Laplace and Z-transforms. However, the Gamma function ${ }^{4}$ was formalized by Harrison in early 2014. In the meantime, we formalized the basic theories of the Laplace transform 36] and the Z-transform [35. Currently, we are working on three main topics which include: 1) formal proofs of the uniqueness of Laplace and Z-transforms which are required to formally verify the inverses of these transforms; 2) vectorial Z-transform, which extend the simple Z-transform over complex vectors; and 3) fractional difference equations, which are mainly based on Gamma function, infinite summations and products over complex functions. Finally, we outline the major tasks to achieve the future milestones as follows:

- Formalization of R-Transform.

- Formalization of Differintegrals for complex-valued functions. This is mainly the generalization of the formalization which was developed in HOL4.

- Formalization of linear fractional differential and difference equations with support to analytical solutions using the transform methods.

During the course of this project, two master and two PhD students have contributed to the formalization. Interestingly, all of them are mainly electrical

4 https://code.google.com/p/hol-light/source/browse/trunk/Multivariate/ gamma.ml 
engineers without prior background of formal methods and higher-order-logic theorem proving. Given the complexity and interdisciplinarity of this research project, it is quite encouraging to see people with an engineering (or physics) background to use proof assistants as a complementary tool. The formalization of the fractional calculus is quite challenging as it requires advanced mathematical concepts of vector integration and Lebesgue measurable functions, etc. So expertise in formal reasoning about these complex mathematical phenomena is required for this formalization, which is quite unique compared to reasoning about software and digital hardware systems. The learning curve of HOL Light varies from student to student. Generally, students start proving basic math equations after a couple of months and the pace of formalization increases over time. Learning HOL Light libraries is not difficult once the basic concepts have been grasped by the user. The formalization of the improper integrals, the Gamma function, the fractional calculus, the Z-transform and the Laplace transform is approximately 15,000 lines of HOL Light code. One of the major obstacles in the formalization was the identification of suitable mathematical definitions and models. Sometimes, textbook proofs do not follow due to various reasons (corner cases, or the proof steps are too abstract, etc.) and they needed to be re-proved on paper with subtle details. Consequently, we have to modify the definitions and thus change the proofs. But now the current formalization seems quite stable as most of the classical properties have been formally verified for our definitions. We believe that future developments can be built on the foundations that have been formalized as most of the work is for general systems. Finally, another important aspect of this project is the potential to apply developed theories to various applications other than fractional calculus. For example, we demonstrated the use of the Gamma function in probability theory [34, the Z-transform in signal processing [35], and the Laplace transform in power electronics 36 .

\section{Conclusion}

In this paper, we mainly presented the motivation and ongoing activities of our long term project about the formalization of fractional calculus in the HOL Light theorem prover. The main contribution of this project is a comprehensive framework of formal definitions and theorems about fractional calculus which can be used to verify modern control, signal processing and electromagnetic systems. Some future directions and recommendations for HOL Light are the improvements in the visualization of proofs, better automation and more accessible tutorials with examples from different engineering/physics topics.

\section{References}

1. N. Pariz A. Kiani-B, K. Fallahi and H. Leung. A Chaotic Secure Communication Scheme Using Fractional Chaotic Systems Based on an Extended Fractional Kalman Filter. Communications in Nonlinear Science and Numerical Simulation, 14:863-879, 2009. 
2. S. K. Afshar, U. Siddique, M. Y. Mahmoud, V. Aravantinos, O. Seddiki, O. Hasan, and S. Tahar. Formal Analysis of Optical Systems. Mathematics in Computer Science, 8(1):39-70, 2014.

3. F. M. Atici. A Transform Method in Discrete Fractional Calculus. International Journal of Difference Equations, 2(2):165-176, 2007.

4. F. M. Atici and P. W. Eloe. Initial Value Problems in Discrete Fractional Calculus. Proceeding of the American Mathematical Society, 137(3):981-989, 2009.

5. T. J. Auastasio. The Fractional-Order Dynamics of Brainstem VestibuloOculomotor Neurons . Biological Cybernetics, 72(1):69-79, 1994.

6. E. Cuestab C. Quintanoa. Improving Satellite Image Classification by Using Fractional Type Convolution Filtering. International Journal of Applied Earth Observation and Geoinformation, 12(4):298-301, 2010.

7. Y. Q. Chen, D. Xue, and H. Dou. Fractional Calculus and Biomimetic Control. In Robotics and Biomimetics, pages 901 -906. IEEE, 2004.

8. M. Marín D. M. Domínguez and M. Camacho. Macrophage Ion Currents are Fit by a Fractional Model and Therefore are a Time Series with Memory . European Biophysics Journal, 38(4):457-464, 2009.

9. M. Dalir and M. Bashour. Application of Fractional Calculus. Applications of Fractional Calculus in Physics, 4(21):12, 2010.

10. S. Das. Functional Fractional Calculus for System Identification and Controls. Springer, 2007.

11. Fernando B. M. Duarte and José António Tenreiro Machado. Pseudoinverse Trajectory Control of Redundant Manipulators: A Fractional Calculus Perspective. In International Conference on Robotics and Automation, pages 2406-2411. IEEE, 2002.

12. S. Elaydi. An Introduction to Difference Equations. Springer, 2005.

13. N. Engheta. Fractional Curl Operator in Electromagnetics. Microwave Optics Technology Letters, 17(2):86-91, 1998.

14. M. Faryad and Q. A. Naqvi. Fractional Rectangular Waveguide. Progress In Electromagnetics Research, PIER, 75:383-396, 2007.

15. G. Gonthier, A. Asperti, J. Avigad, Y. Bertot, C. Cohen, F. Garillot, S. Le Roux, A. Mahboubi, R. OConnor, S. Ould Biha, I. Pasca, L. Rideau, A. Solovyev, E. Tassi, and L. Thry. A Machine-Checked Proof of the Odd Order Theorem. In Interactive Theorem Proving, volume 7998 of LNCS, pages 163-179. Springer, 2013.

16. T. C. Hales. Introduction to the Flyspeck Project. In Mathematics, Algorithms, Proofs, volume 05021 of Dagstuhl Seminar Proceedings, pages 1-11, 2005.

17. T. T. Hartley and C. F. Lorenzo. Fractional System Identification: An Approach Using Continuous Order Distributions. Technical report, National Aeronautics and Space Administration, Glenn Research Cente NASA TM, 1999.

18. W.M. Ahmad K. Assaleh. Modeling of Speech Signals Using Fractional Calculus. In International Symposium on Signal Processing and its Applications, pages 1-4. IEEE, 2007.

19. B. T. Krishna and K. V. V. S. Reddy. Design of Digital Differentiators and Integrators of Order $\frac{1}{2}$. World Journal of Modelling and Simulation, 4:182-187, 2008.

20. G. W. Leibnitz. Leibnitzens Mathematische Schriften. SIGDA News Letter, 2:301302, 1962.

21. K. A. Lurie. An Introduction to the Mathematical Theory of Dynamic Materials. Springer, 2007.

22. Richard L. Magin. Fractional Calculus Models of Complex Dynamics in Biological Tissues. Computers and Mathematics with Applications, 59:1586-1593, 2010. 
23. G. Maione and P. Lino. New Tuning Rules for Fractional $p i^{\alpha}$ Controllers. Nonlinear Dynamics, 49(1-2):pp 251-257, 2007.

24. K. S. Miller and B. Ross. An Introduction to Fractional Calculus and Fractional Differential Equations. John Willey, 1993.

25. Q. A. Naqvi and M. Abbas. Complex and Higher Order Fractional Curl Operator in Electromagnetics . Optics Communications, 241:349-355, 2004.

26. K. Ogata. Modern Control Engineering. Prentice Hall, 2010.

27. K. B. Oldham and J. Spanier. The Fractional Calculus. New York, Academic Press, 1974.

28. A. V. Oppenheim, R. W. Schafer, and J. R. Buck. Discrete-Time Signal Processing. Prentice Hall, 1999.

29. B. Mathieu, P. Melchior, A. Oustaloup and Ch. Ceyral. Fractional Differentiation for Edge Detection. Signal Processing, 83:2421-2432, 2003.

30. I. Petrás and B.M. Vinagre. Practical Application of Digital Fractional-Order Controller to Temperature Control. Acta Montanistica Slovaca, 7(2):131-137, 2002.

31. I. Podlubny. Fractional Differential Equations, Academic Press. 1999.

32. B. Ross. A Brief History And Exposition of The Fundamental Theory of Fractional Calculus. In Fractional Calculus and Its Applications, volume 457 of Lecture Notes in Mathematics, pages 1-36. Springer, 1975.

33. U. Siddique and O. Hasan. Formal Analysis of Fractional Order Systems in HOL. In Formal Methods in Computer Aided Design, pages 163-170. IEEE, 2011.

34. U. Siddique and O. Hasan. On the Formalization of Gamma Function in HOL. Journal of Automated Reasoning, 53(4):407-429, 2014.

35. U. Siddique, M. Y. Mahmoud, and S. Tahar. On the Formalization of Z-Transform in HOL. In Interactive Theorem Proving, volume 8558 of LNCS, pages 483-498. Springer, 2014.

36. S. H. Taqdees and O. Hasan. Formalization of Laplace Transform Using the Multivariable Calculus Theory of HOL-Light. In Logic for Programming, Artificial Intelligence, and Reasoning, volume 8312 of LNCS, pages 744-758. 2013.

37. C. C. Tseng. Design of Fractional Order Digital FIR Differentiators. IEEE Signal processing Letters, 8(3):77-79, 2001.

38. B. M. Vinagreb Y. Q. Chena. Fractional Differentiation for Edge Detection. Signal Processing, 83:2359-2365, 2003.

39. X. S. Yang. Mathematical Modeling with Multidisciplinary Applications. John Wiley, 2013.

40. V. Zaborovsky and R. Meylanov. Informational Network Traffic Model Based on Fractional Calculus. In Proceedings of the International Conference Info-tech and Info-net, pages 58-63. IEEE, 2001. 